\title{
A criança éo pai do homem: novos desafios para a área de saúde da criança
}

\author{
Child is the father of man: new challenges for child health
}

M aria Elisabeth Lopes M oreira ${ }^{1}$

M arcelo Zubaran Goldani ${ }^{2}$

\footnotetext{
${ }^{1}$ Instituto Fernandes Figueira, Fiocruz. bebeth@iff.fiocruz.br ${ }_{2}^{2}$ Universidade Federal do Rio Grande do Sul.
}

Abstract Thispaper offersan analysis of current demographic and epidemiological context in which the child falls and addresses the need to the reformulation of the health services intended for child care, pointing also to the question of the need to produce new knowledge that help them in a new pediatricscare. Theknowledgethat child care needs to be expanded to take into account acute problems and disease prevention in the long run, even in adulthood, introduces a new field for pediatrics that also demands the formation of new researchers who can give the children opportunities to be included in research on drugs and other therapeutic proposals addressed to them. In conclusion, health care strategies concentrated particularly on children with all their specificity, a growing and developing human being, need to be implemented in the health services so that they can grow well and become healthy adults.

Key words Pediatrics, Child care, Demography, Research
Resumo Este artigo traz uma análise do atual contexto epidemiológico e demográfico onde a criança se insere e trata da necessidade de reformulação dos servi ços de saúde destinados ao cuidado a criança, apontando também para a questão de que é necessária a produção de novos conhecimentos que dêem conta de uma nova pediatria. 0 conhecimento de que o cuidado aplicado à criança precisa ser ampliado de forma a contemplar problemas agudos e prevenção de doenças a longo prazo, inclusive na vida adulta, introduz um novo campo de ação para a pediatria, exigindo também a formação de novos pesquisadores que dêem às crianças oportunidades de serem incluídas em pesquisas com medicamentos e outras propostas terapêuticas a elas endereçadas. Concluindo, novas estratégias relacionadas ao cuidado à criança e à formação de novos pediatras e pesquisadores, que dêem conta do cuidado de um ser humano em crescimento e desenvolvimento, com todas as suas especificidades, precisam ser implementadas nos serviços de saúde e no campo da produção de conhecimentos, para que estas possam crescer e se tornar adultos saudáveis.

Palavras-chave Pediatria, Cuidado da criança, Demografia, Pesquisa 
Introdução

N os últimos anos, o perfil das taxas de mortalidadeinfantil tem mudado substancialmente. As políticas públicas desaúde, endereçadas ao tratamento precoce das pneumonias ediarréias, associadas a um grande esforço na prevenção das doenças infecciosas por meio das vacinações, contribuíram significativamente para a redução da mortalidade entre os dois meses e cinco anos de vida, propondo novos desafios a serem enfrentados através da produção de novos conhecimentos.

O novo cenário estabel ecido no campo da saúde da criança e do adolescente provocou o surgimento de um campo específico da pesquisa biomédica, consequência de um processo de transição epidemiológica e demográfica, ocorrido ao longo do último século. Inicialmente, nos países desenvolvidos, a melhoria das condições sociais, levando ao arrefecimento das taxas de mortalidade infantil, redução das taxas de fertilidade e o envelhecimento das populações, determinou um novo padrão de saúde doença, no qual as taxas de enfermidade agudas e infecciosas foram suplantadas em termos de prevalência por moléstias crônicas e degenerativas ${ }^{1}$. N este sentido, os indivíduos passaram a viver por mais tempo, porém, em contrapartida, passaram a ficar mais tempo doentes. $\mathrm{Na}$ esteira desse intenso processo de transição demográfica e epidemiológica, a elaboração de novas linhas de causalidade foi necessária para tentar esclarecer as novas associações entre eventos precoces e desfechos muito tardios.

No Brasil, esse mesmo processo de transição desenrolou-se timidamente até a metade do sé culo passado, apresentando um revés si gnificativo ao longo do período da ditadura militar, em consequência da redução dos investimentos sociais e da renda média familiar. A partir de meados da década de setenta, com a mel hora paulatina das condi ções sociais do país ea redução do preço de alimentos, notou-se uma retomada da queda da mortalidadeinfantil, principalmentena última década do século passado ${ }^{2}$. Ao mesmo tempo, ocorreu uma elevação da expectativa de vida, seguindo uma tendência há muito identificada nos países industrializados.

Nota-se também nesse período o início da redução significativa das taxas de desnutrição, gradativamente substituída por um aumento nas taxas de sobrepeso e obesidade em todos os estratos etários ${ }^{3,4}$. Esse fenômeno, adjacente ao processo de transição epidemiológica e demográfica, denominado como transição nutricional, caracteriza-se pela rápida mudança do pa- drão nutricional da população humana, diretamente associada às modificações na quantidade e qualidade da dieta, relacionando-se com mudanças econômicas, demográficas, ambientais e culturais ocorridas nas sociedades nos últimos quarenta anos3,4. No Brasil, a elevação do nível de escolaridade materna, aumento do poder de compra das famílias e ampliação do acesso aos cuidados desaúde foram determinantes para essa mudança 5 .

Todavia, seria importante salientar que essa série de fenômenos ocorridos nas últimas décadas no mundo foi permeada por características sociais peculiares a cada país. No caso do Brasil, esse processo sofreu a influência do alto grau de desigualdades em saúde, produzindo um vasto número de diferenças no desenvolvimento desse fenômeno. Assim sendo, diferenças geográficas, em relação ao gênero eàs gerações acentuam uma grande diversidade de aspectos desse processo de transição. Mulheres teriam a propensão de serem menos obesas, as classes mais privilegiadas apresentariam maior chance de obesidade, a redução de mortalidade infantil e a fertilidade ocorreriam preferencialmente em localidades e locais mais desenvolvidos. Enfim, longe de se apresentar como um processo homogêneo, a transição demográfica epidemiológica determinou, em alguns casos, um aumento das diferenças e desigualdades em relação a alguns desfechos. Em outros, inversamente, produz similaridades, em consequência da utilização indiscriminada de novas tecnologias versus a escassez de acesso às mesmas ${ }^{6}$.

\section{A criança \\ no contexto da transição epidemiológica}

Em relação à criança, tornaram-se necessárias, também, mudanças nos modelos de atenção à saúde. M odelos hospitalocêntricos não são mais suficientes para dar conta da "nova pediatria" que as demandas e os conhecimentos atuais vêm impondo. Desafios relacionados a tratamento e prevenção de doenças impõem-se como fortes demandas para a criação deum novo modelo de cuidado para garantir a saúde de um indivíduo em crescimento e desenvolvimento. N este sentido, a integração dos novos conhecimentos produzidos com os avanços tecnológicos e a nova demografia que se apresenta aponta para um modelo de cuidado ampliado, visando à prevenção das doenças infantis e, também agora, das doenças do adulto. 
Os resultados da Pesquisa Nacional de Demografia e Saúde da Criança e da Mulher de 2006 demonstram uma diminuição, em números absolutos e relativos, de crianças nas faixas etárias de 0 a 4 e 5 a 9 anos, bem como uma redução da taxa de fecundidade para níveis em torno de 1,8 filhos/mulher associado a um aumento da expectativa de vida da população brasileira ${ }^{7}$. Estes indicadores apontam para a necessidade de reorganização dos serviços de saúde, organizados atualmente em torno do cuidado agudo ao adulto, com baixo investimento para a prevenção precoce das doenças crônico-degenerativas ainda na infância. $A$ Tabela 1 apresenta as diferenças entre os indicadores de saúde em dez anos apontados pelos resultados da Pesquisa Nacional de Demografia e Saúde da Criança eda Mulher (PNDS), 1996 e 20067,8.

0 fato é que há uma nova realidade relacionada à saúde da criança no mundo inteiro, com boase más novidades. A mortalidadeinfantil caiu, as taxas de aleitamento materno aumentaram, 0 acesso às vacinas aumentou, assim como 0 acesso ao pré-natal. Por outro lado, o número de partos prematuros aumentou e também a incidência de obesidade. Outra questão que se apresenta é 0 aumento do número de crianças com doenças crônicas. 0 acesso às tecnologias modernas e sofisticadas permitiu o aumento da sobrevida de crianças que antes não sobreviveriam - pré-termos extremos, portadores de malformações congênitas e doenças crônicas - , criando uma nova clientela que precisa ser atendida nos serviços de saúde e que demanda um alto nível de cuidado da sociedade: a criança dependente detecnologia.
Atualmente, nos países desenvolvidos, 90\% das crianças nascidas com doenças crônicas alcançam a idade adulta. 0 aumento significativo de crianças com necessidades especiais em saúde (CNES), que demandam um alto grau de cuidados ao longo da vida, produziu pelo menos dois fenômenos importantes na área da assistência à saúde e de administração de recursos ${ }^{9}$. Em primeiro lugar, o diagnóstico precoce de doenças potencialmente crônicas ou a sobrevivência de crianças com sequelas permanentes determinaram uma especificidade de assistência à saúde dedicada a prestar cuidados por longos períodos, muitas vezes durante todo o ciclo vital do indivíduo, em um modelo que deveser necessariamenteclínico-desenvolvimentista. Em segundo lugar, mudanças epidemiológicas e demográficas suscitam questões relacionadas à administração de recursos humanos e financeiros, promovendo a necessidade de implantação de novas estratégias de assi stência e aperfeiçoamentos do sistema de saúde.

\section{As peculiaridades da infância que impactam na atenção à saúde}

A infância é um período único na vida e esteperíodo tem fortes influências na vida adulta. Forrest et al. ${ }^{10}$, em um artigo publicado no JAM A em 1997 para explicar por quea infância éum período fundamental da vida humana, descrevem quatro características que a distinguem (4 Ds): 1) desenvolvimento (a criança é um ser em desenvolvimento e sua saúde depende do seu padrão decrescimento edesenvolvimento em geral

Tabela 1. Diferenças entre os indicadores de saúde em dez anos.

\begin{tabular}{lrr}
\hline & 1996 & 2006 \\
\hline Taxa de fecundidade \% & 2,5 & 1,8 \\
Taxa de fecundidade entre 15-19 anos \% & 17 & 23 \\
Pré-natal com sete ou mais consultas & 47 & 61 \\
M ulheres que receberam vacina contra tétano na gravidez \% & 64 & 70 \\
Taxa de cesárea \% & 36 & 44 \\
Prevalência de desnutrição na infância \% & 13,5 & 6,8 \\
Obesidade na infância \% & 4,9 & 7 \\
Aleitamento materno exclusivo entre 1-3 meses \% & 40 & 45 \\
M ortalidade infantil & $39 / 1000$ & $22 / 1000$ \\
Duração mediana para aleitamento materno & 1 mês & 2,2 meses \\
\hline
\end{tabular}

Fonte: PNDS 1996 e $2006^{7,8}$ 
e em especial do desenvolvimento cognitivo e emocional); 2) dependência (as crianças dependem de um cuidador para que tenham acesso aos cuidados à saúde e em especial à prevenção às doenças); 3) diferenças (crianças apresentam um aspecto epidemiológico diferente em relação à presença da doença e aos riscos de sequelas, ou seja, são muito mais vulneráveis aos insultos); 4) demografia (o perfil demográfico das crianças também é diferente; as mais vulneráveis vivem em condições de maior pobreza, com me nos acesso aos serviços de saúde, o que poderia dar origem a um quinto $D: 5$ ) desigualdade.

0 cuidado às crianças necessariamente envolvecaracterísticas deseu desenvolvimento, que se não forem abordadas afetarão sua saúde e desempenho na vida adulta e, portanto, a sociedadeem quevivem. Vigilância relacionada a questões anteriormente pouco valorizadas nos serviços de saúde, como oportunidades de aprendizagem, monitoramento dealterações de comportamento, prevenção deacidentes, identificação de violência familiar e abusos domésticos, é fundamental para a promoção da saúde e parte importante do adulto queno qual a criança se transformará. A criança éum ser em crescimento, em direção a uma vida adulta saudável e, por isto, alteraçõesno seu processo decrescimento/desenvolvimento vêm sendo utilizadas ao longo do tempo no diagnóstico das condições de saúde de uma dada população. Este conceito já éutilizado desde a vida fetal, na medida em que os obstetras se utilizam da avaliação do crescimento intrauterino como indicador de bem- estar fetal. Além disto, a média da estatura final das populações ou subpopulações é usada como proxy das condições socioeconômicas de regiões e países em diferentes épocas da história. São bem conhecidos no meio acadêmico e pediátrico os estudos de Engels sobre o déficit de crescimento das crianças inglesas que trabalhavam em fábricas na época da revolução industrial eos deStern, comprovando o impacto do racionamento alimentar por ocasião da segunda guerra nas condições de saúde a longo prazo na coorte da H olanda ${ }^{11}$.

Outros estudos mais atuais demonstram o impacto da restrição do crescimento intra-uterino no desenvolvimento da síndrome metabólica do adulto (hipercolesterolemia, diabetes, hipertensão eobesidade) ${ }^{12}$ ea hipótese dequeinsultos que levam à alteração do crescimento em fases precoces da vida (programming) podem resultar em déficit permanente ou a longo prazo na estrutura ou função do organismo vem sendo investigada por vários pesquisadores ${ }^{13}$.
A criança é o pai do homem: desafios eoportunidades

A criança é o pai do homem e não importa que tenha sido Wordsworth ou Freud o autor da frase. As condições de saúde no início da vida são fortes determinantes da saúde do adulto e isto não tem merecido atenção suficiente ainda nos dias de hoje. Entretanto, os novos conceitos trazidos pela epigenética sobre como as interações entregenes eambiente podem modificar um traço ou uma predisposição genética sem alterar o genoma têm merecido atenção e devem modificar o olhar sobre a criança. Epigenética é um campo da biologia que estuda as interações causais entre genes e seus produtos que são responsáveis pela produção do fenótipo. Assim sendo, a história de vida dos sujeitos, começando na infância com o padrão nutricional, decrescimento ehábitos de vida, pode influenciar o fenótipo do individuo e, consequentemente, sua qualidade de vida futura e isto pode mudar o olhar sobre a saúde da criança ${ }^{14}$.

Nessecenário, a teoria da origem desenvolvimentista da saúde e da doenças ( $\mathrm{DOH}$ aD, do inglês developmental origins of health and disease), sustentada inicialmente por uma série de axiomas e após por demonstrações empíricas e experimentais, surge como uma área de pesquisa dedicada a esclarecer as relações entre eventos ocorridos em fases precoces do desenvolvimento e determinados padrões de doença e saúde ao longo da vida. A construção de novos conhecimentos advindos dessa área foram oferecidos para demarcar mudanças tanto de ordem financeira organizacional como também em termos de políticas públicas na área da saúde e do bem-estar social ${ }^{15-17}$.

$\mathrm{O}$ conceito de $\mathrm{DOH} \mathrm{aD}$ surgiu da idéia original desenvolvida por Barker e denominada origem fetal da doença do adulto (FOAD, do inglês fetal origins of adult disease) ${ }^{17}$. Contudo, resultados de vários estudos evidenciaram que o risco para o desenvolvimento de doenças crônicas na fase adulta da vida advinha não apenas de eventos precoces da vida fetal, porém também detodo o período de desenvolvimento e ao longo da infância e adolescência. Deoutro modo, a inclusão do termo "saúde" refere-se ao compromisso dessa área com o desenvolvimento de estratégias objetivando à prevenção de moléstias crônicas.

$M$ ais recentemente, outra área de pesquisa adjacente, a "biologia evolucionária desenvolvimentista" (evo-devo, do inglês evolutionary developmental biology), contribuiu para alargar 0 
conceito $\mathrm{DOH}$ aD por meio de novos conhecimentos. Essa área oferece a demonstração da presença defatores preconcepcionais capazes influenciar o padrão de saúde e doença, adquiridos pela adoção de estratégias adaptativas, de senvolvidas por meio de um processo evolucionário. Esses fatores determinariam o grau da capacidade biológica do ser adequar-se ao ambiente existente ou a mudanças ocorridas nele. Como exemplo, atrasos de crescimento somáticos e recuperação posterior podem ser consideradas respostas adaptativas a eventos de crises de energia. N esse caso, a capacidade desenvolvida ao longo de gerações para mediar o dispêndio e 0 armazenamento de energia de acordo com a afluência de recursos possibilitou a superação de tempos de escassez. Contudo, em momentos de pletora, poderia conferir efeitos adversos, tais como a obesidade ${ }^{18}$.

Outro mecanismo crucial envolvido dentro do conceito $D O H$ aD éo efeito epigenético determinado por influências ambientais, promovendo o controle do processo de transcrição dos genes de modo seletivo. Esse mecanismo faz parte do cabedal de ferramentas adaptativas necessário para suplantar eventos adversos. Porém, uma vez que esse mecanismo seja alterado, esta mudança pode induzir o surgimento de doenças epigenéticas, tais como tumores raros em humanos $^{19}$.

\section{0 investimento na atenção à criança ena produção de novos conhecimentos}

Pouco se tem investido, no mundo inteiro, na produção de novos conhecimentos na área da saúde da criança, apesar do fato conhecido de que o investimento na saúde da criança pode proporcionar melhores condições de saúde para 0 adulto ${ }^{20}$.

O uso de medicações "off-label" ainda é uma questão importantee indesejável na área da saúde da criança e são várias as razões para exclusão das crianças no campo do desenvolvimento de novas drogas: questões éticas, dificuldades técnicas e logísticas ligadas à farmacodinâmica/farmacocinética ebiodisponiblidade das drogas que são idade-dependentes e questões econômicas. N os últimos anos, a criança tem sido considerada como órfã terapêutica e, em geral, os tratamentos a ela dispensados são baseados em experiências pessoais, relatos de casos, recomendações de especialistas ou ensaios clínicos pequenos que não podem ser generalizados para um grande número de crianças. A proximadamente dois terços das medicações usadas na prática clínica pediátrica não possuem evidências suficientes sobre dosagens, segurança e eficácia para cada período do desenvolvimento ${ }^{21,22}$.

0 número de estudos clínicos randomizados em crianças é reconhecidamente pequeno quando comparado ao número de estudos em adultos e algumas hipóteses têm sido propostas para explicar este fenômeno. Os pesquisadores e os comitês de ética estão expostos a um duplo desafio: 0 deassegurar à criança o direito deter acesso aos estudos randomizados e, ao mesmo tempo, garantir que os riscos das pesquisas nelas realizadas sejam minimizados. Uma outra questão é a dificuldade no recrutamento de crianças para participação em estudos clínicos, algumas vezes pela relutância dos pais e outras pela baixa prevalência de uma determinada condição clínica em uma dada idade. Além destas razões, a escassez de investimentos na área dificulta a realização de estudos nesta faixa etária, o que também prejudica a formação denovos pesquisadores eo suportetécnico necessário para os atuais²2.

$\mathrm{Na}$ área da pesquisa pediátrica, estamos vivendo atualmente com uma dramática escassez de recursos. Sabemos, por exemplo, que somente $4,4 \%$ do orçamento do $\mathrm{N}$ ational Institutes of $\mathrm{H}$ ealth foi destinado ao $\mathrm{N}$ ational Institute of Child $\mathrm{H}$ ealth and $\mathrm{H}$ uman Development ${ }^{20}$. No Brasil, este fato não é diferente e embora não tenhamos dados específicos em relação ao montante dos financiamentos das agências de fomento dirigidos à pesquisa na área da saúde da criança, sabemos que em geral estes são escassos.

A visibilidadeglobal desta escassez de investimento ainda é modesta, bem como o entendimento de que este investimento podesignificar a preservação da saúde a longo prazo. Para dar um exemplo, sabemos que a prevalência de obesidade na infância vem aumentando e em al guns locais já chega a cerca de $30 \%{ }^{23}$. Se não houver um investimento maciço para o entendimento deste problema e em ações que possam reverter este quadro, esta geração de crianças será a primeira em dois séculos que terá uma expectativa de vida menor do que a de seus pais ${ }^{20}$.

\section{Consideraçõesfinais}

Os novos indicadores apontados pelo último PNDS em 2006 têm mobilizado os gestores em saúde para uma necessária reorganização de serviços que dêem conta do envelhecimento da po- 
pulação nesta nova demografia que se apresenta. Entretanto, 0 ol har não pode se desviar da criança para o idoso simplesmente. Existe também neste novo modelo que se apresenta indicativos fortes de queéna infância que se previneà maioria das doenças crônico-degenerativas que vão da hipertensão e diabetes ao câncer na adultice. A criança precisa se tornar visível, nas questões que vão do cuidado à sua saúde, considerando suas especificidades, mas também ao se considerar que éna infância que se constrói um adulto saudável. O portunidades perdidas na infância são oportunidades não retornáveis. A apoptose ou morte programada das células é uma realidade. Após a infância, a maioria dos órgãos não vai formar novas células e, portanto, se na infância não se cuidou da criança de forma adequada, ela já começará a vida adulta com um número menor de células em diversosórgãos. Estas células serão mais rapidamente destruídas, alterando a função dos órgãos precocemente, levando a doenças crônicas indivíduos cada vez mais jovens.
Não se pode também fechar os olhos para os acontecimentos perinatais da atualidade. As crianças estão diminuindo porque os índices defertilidade estão diminuindo, mas também porque uma grande parte delas ainda morre na primeira semana de vida. Além disto, as crianças doentes continuam a ser submetidas ao arsenal terapêutico desenvolvido para adultos, sem que nenhum estudo de eficácia ou segurança seja realizado. 0 contingente dos chamados órfãos terapêuticos, portanto, é grande, e uma droga mal usada na infância pode comprometer, por exemplo, a função renal para sempre, predispondo a doenças renais precoces na vida adulta que vão demandar um alto custo dos serviços de saúde. 0 que fazer? Precisamos tornar a criança e suas potencialidades visíveis e prevenir na infância as doenças crônico-degenerativas, para que não precisemos lidar com al tos custos de saúde no futuro, o que poderia inviabilizar o sistema de saúde no qual o acesso é um direito de todos.

\section{Colaboradores}

MEL Moreira e M Z Goldani participaram da concepção, elaboração do artigo e da revisão final do documento. 


\section{Referências}

1. Macfarlane A. Social class variations in perinatal mortality. The journal of maternal and child health 1979; 337:3339-3341.

2. Puffer RR, Serrano CV. Características del peso al nacer [publicación científica 504]. Washington, D.C.: Organización Panamericana de la Salud; 1988.

3. Monteiro CA, Moura EC, Conde WL, Popkin BM Socioeconomic status and obesity in adult populations of developing countries: a review. Bull World Health Organ. 2004; 82:940-946.

4. Popkin BM. Nutrition in transition: the changing global nutrition challenge. Asia Pac J Clin Nutr. 2001;10(Suppl.):S13-18 .

5. Monteiro $\mathrm{CA}$, Benicio $\mathrm{MH}$, Konno SC, Silva $\mathrm{AC}$ Lima $A L$, Conde $W L$. Causes for the decline in child under-nutrition in Brazil, 1996-2007. Rev. Saude Publica 2009; 43:35-43.

6. Silveira PP, Portella AK, Goldani MZ. Obesity in Latin America: similarity in the inequalities. Lancet 2005; 366(9498):451-452.

7. Brasil. Ministério da Saúde. CEBRAP. Pesquisa $\mathrm{Na}$ cional de Demografia e Saúde da Criança e da Mulher PNDS 2006. Dimensões do Processo Reprodutivo e da Saúde da Criança. Brasília: Ministério da Saúde; 2008.

8. Brasil. M inistério da Saúde. BENFAM. Pesquisa Nacional de Demografia e Saúde da Criança e da Mulher PNDS 1996. Dimensões do Processo Reprodutivo e da Saúde da Criança. Brasília: Ministério da Saúde; 1996.

9. Gortmacher SL, Sappenfield W. Chronic childhood disorders: prevalence and impact. Pediatr Clin N orth Am. 1984; 31:3-18

10. Forrest CB, Simpson L, Clancy C. Child Health Services research: Challenges and opportunities. JAM A 1997; 277: 1787-1793.

11. Bogin B. Patterns of human growth. $2^{\text {nd }}$ ed. Cambridge: Cambridge University Press; 1999.

12. Barker DJ. The developmental origins of well-being. Philos Trans R Soc Lond B Biol Sci. 2004; 359(1449): 1359-1366.
13. Lucas A. Long-term programming effects of early nutrition - implications for the preterm infant. J Perinatol. 2005; 25:S2-6.

14. Silveira PP, Portella AK, Goldani MZ, Barbieri MA. Developmental origins of health and disease (DOH aD). J Pediatr (Rio J). 2007; 83:494-504.

15. Sinclair KD, Lea RG, Rees WD, Young LE. The developmental origins of health and disease: current theories and epigenetic mechanisms. Soc Reprod Fertil Suppl. 2007; 64:425-443.

16. Gluckman PD, Hanson MA, Buklijas T, Low FM, Beedle AS. Epigenetic mechanisms that underpin metabolic and cardiovascular diseases. $\mathrm{N}$ at $\mathrm{Rev}$ Endocrinol. 2009; 5(7):401-408.

17. Barker DJ. The origins of the developmental origins theory. I Intern Med. 2007; 261:412-417.

18. Hochberg Z. Evo-devo of child growth II: human life history and transition between its phases. Eur J Endocrinol. 2009; 160(2):135-141.

19. Gluckman PD, Hanson MA, Bateson $P$, Beedle AS, Law CM, Bhutta ZA, Anokhin KV, Bougnères $P$, Chandak GR, Dasgupta P, Smith GD, Ellison PT, Forrester TE, Gilbert SF, Jablonka E, Kaplan H Prentice AM, Simpson SJ, U auy R, West-Eberhard MJ. Towards a new developmental synthesis: adaptive developmental plasticity and human disease. Lancet 2009; 373(9675):1654-1657.

20. Shaul PW. The cold, hard truth about pediatric research. Pediatric Research 2007; 61:506-509.

21. Ward RM, Kauffman R Future of Pediatric Therapeutics: reauthorization of BPCA and PREA. Clinical Pharmacology \& Therapeutics 2007; 81:477-479.

22. $M$ artinez- $C$ astaldi $C$, Silverstein $M$, Bauchner $H$. Child versus adult research: the gap in high quality study design. Pediatrics 2008; 122:52-57.

23. Dunton GF, Kaplan J, Wolch J, Jerrett M, Reynolds KD. Physical environmental correlates of childhood obesity: a systematic review. O bes Rev. 2009;10:393402. 